\title{
Ability to successfully use an epinephrine auto-injector after switching to a different device
}

\author{
Robert Boyle*, Annabella Procktor, Katherine Phillips, Camila Pinto, Heather Hanna, Thisanayagam Umasunthar
}

From Food Allergy and Anaphylaxis Meeting 2014

Dublin, Ireland. 9-11 October 2014

\section{Rationale}

Patients previously prescribed one epinephrine autoinjector device may be switched to an alternative device by their pharmacist or physician - sometimes without training on the new device. It is unclear whether "device switches" without retraining compromise the ability to deliver epinephrine.

\section{Methods}

We evaluated mothers of food-allergic children participating in a UK study of epinephrine auto-injectors (EAI), 1 year after they were first trained to use an EAI, either Anapen or Epipen (old design). Participants' ability to deliver epinephrine using their device was assessed using a simulated anaphylaxis scenario. Participants then underwent repeat assessment using a different EAI device, randomly allocated, without training on the new device. The UK-approved EAIs Epipen (new/old designs), JEXT or Anapen were used, or Intelliject, an EAI with audio/visual prompts approved in North America as AuviQ(tm) and Allerject ${ }^{\mathrm{TM}}$. ISRCTN29175528

\section{Results}

We evaluated ability to deliver epinephrine in 108 participants. Overall success rates were similar using their original EAI 68/108 (63\%) to the new device 65/108 $(60 \% ; \mathrm{P}=0.775)$. However the outcome differed significantly for different types of device switch. Success rates were lower when switching between Anapen and either old Epipen, new Epipen or JEXT (6/18; 33\%) compared with switching from old Epipen to either new Epipen or JEXT (30/42; 71\%; $\mathrm{P}<0.009)$. Success rates were highest when switching from Anapen or old Epipen to Intelliject $(26 / 28$; 93\%) compared with switching to other EAIs (39/80; 49\%; $\mathrm{P}=0.000)$.

Imperial College London, London, UK 